Water Management

Volume 169 Issue WM1

Method for assessing discharge in meandering compound channels

Liu, Shan, Liu and Yang
Proceedings of the Institution of Civil Engineers Water Management 169 February 2016 Issue WM1 Pages 17-29 http://dx.doi.org/10.1680/wama.14.0013 Paper 1400131 Received 15/11/2014 Published online 20/03/2015 Accepted 26/01/2015 Keywords: fluid mechanics/mathematical modelling/river engineering

ICE Publishing: All rights reserved ice

Institution of Civil Engineers

\title{
Method for assessing discharge in meandering compound channels
}

1 Chao Liu BSC

PhD Candidate, State Key Laboratory of Hydraulics and Mountain River Engineering, Sichuan University, Sichuan, China; College of Water Resource and Hydropower, Sichuan University, Sichuan, China

2 Yuqi Shan BSC

PhD Candidate, College of Mathematics, Sichuan University, Sichuan, China
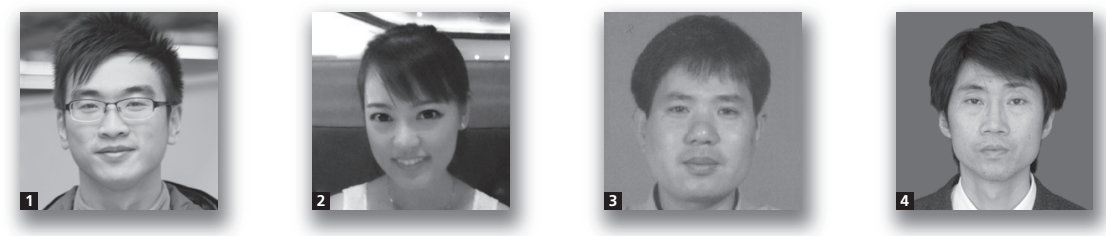

Laboratory experiments were conducted in a large-scale meandering compound channel to investigate the flow patterns at the apex and cross-over sections. An equation in curvilinear co-ordinates was derived from the momentum equation and the flow continuity equation. In this equation, two shear stress terms are ignored due to their negligible contributions such that simple expressions are obtained. Several groups of experimental data from the published literature were used to verify this model. Comparison of the experimental and predictive results indicates that the proposed method is capable of accurately forecasting the stage discharge in meandering compound channels. Finally, the simple analytical solution of this model and the velocity parameter are further discussed.

\section{Notation}$$
B
$$$$
B_{\mathrm{m}}
$$$$
b
$$$$
\mathrm{C}_{\mathrm{p}}
$$$$
D_{\mathrm{r}}
$$$$
F_{\mathrm{c}}
$$$$
f
$$$$
g
$$$$
\text { H }
$$$$
h
$$$$
k
$$$$
n
$$

$Q$

$Q_{\text {fp }}$

$Q_{\mathrm{mc}}$

$q$

$R$

$R_{\mathrm{h}}$

$R_{\mathrm{m}}$

$r$ total channel width

meander belt width

meandering main channel width

contribution percentage

relative flow depth, defined as $D_{\mathrm{r}}=(H-h) / H$

centrifugal force

Darcy-Weisbach friction factor

local gravitational acceleration

flow depth

bankfull depth

dimensionless coefficient reflecting the relation

between secondary flows and $F_{\mathrm{c}}$

Manning's coefficient

total discharge

floodplain discharge

main channel discharge

local unit discharge, defined as $q=\int_{0}^{H} U \mathrm{~d} z$

outer radius

hydraulic radius

medium radius

inner radius

bed slope in Equation 1

valley slope

meandering main channel slope, defined as

$S_{\mathrm{mc}}=S_{\mathrm{fp}} / S$

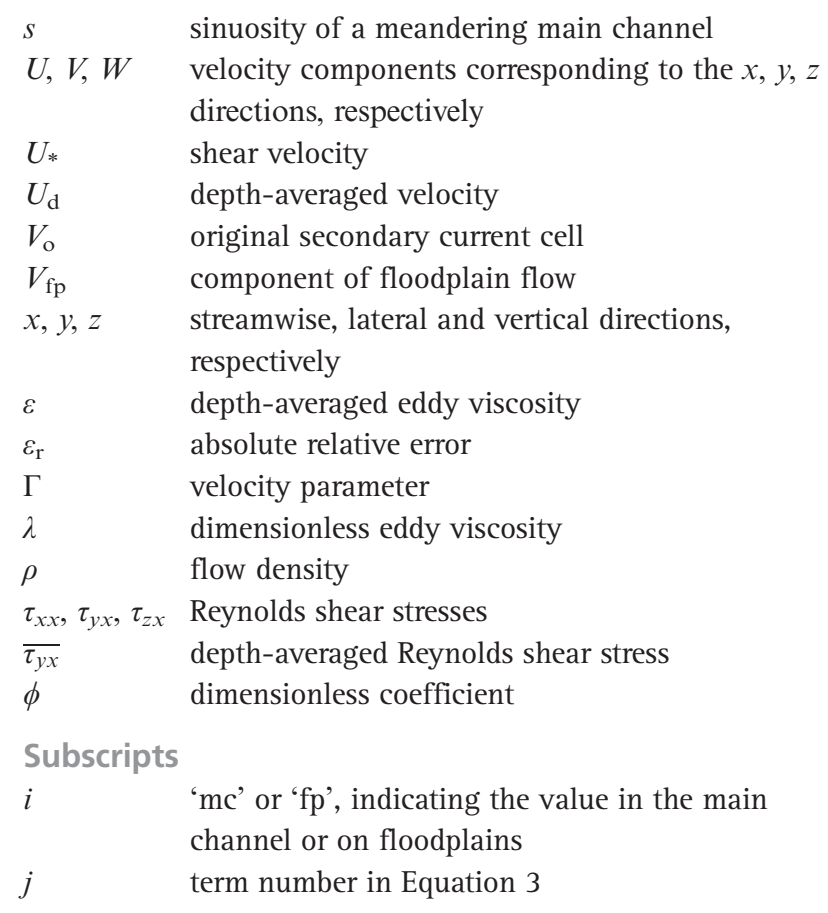

\section{Introduction}

A meandering channel is often observed in natural alluvial rivers, and the curved main channel balances sediment transport 
and bed erosion. When a flood occurs, the floodplains aid the meandering main channel to convey the extra water downstream such that a meandering compound channel is observed. The flow characteristics in the meandering compound channel are different from those in the straight channel. In a straight channel, a velocity difference is observed between the main channel flow and the floodplain flow, which causes strong lateral shear stresses in the mixing region. In a meandering channel, the flow under the bankfull level prefers to progress downstream in the meandering main channel, but the floodplain flow travels along the valley direction (Shiono and Muto, 1998), which causes strong vertical shear stresses near the bankfull level in the cross-over sections. Owing to the effects of centrifugal force and upstream flood plain flow, the secondary flow in the meandering main channel is strengthened, which may affect lateral sediment transport and longitudinal bed morphology (Lyness et al., 2001; Shiono et al., 2008, 2009; Spooner, 2001).

The stage-discharge curve is important in hydraulic engineering projects. With consideration of the complicated flow interaction between the meandering main channel flow and the floodplain flow, many methods have been presented for predicting the stage-discharge curve (Greenhill and Sellin, 1993; Patra and Kar, 2000; Patra et al., 2004; Shiono et al., 1999) based on the divided panel method and the classical Manning's equation. However, the predictive capabilities of these methods were not satisfactory compared with the performance of these methods in straight channels. In addition, several two-dimensional (2D) and three-dimensional (3D) numerical models offer high-accuracy modelling performance in the curved channel (Guo et al., 2008; Jing et al., 2009; Morvan et al., 2002; Shao et al., 2003; Zarrati et al., 2005). Also, certain 2D analytical methods that model the lateral distribution of depth-averaged velocity at the apex section have been presented (Ervine et al., 2000; Huai et al., 2009; McGahey and Samuels, 2003) and, recently, a 2D analytical method capable of predicting lateral velocity profiles along a meander was proposed (Liu et al., 2014a). These models indicate that modelling work in a meandering channel is difficult because additional factors must be considered (Ervine et al., 1993). In addition, curvilinear co-ordinates are more suitable for the meandering channel than vertical co-ordinates (Schlichting, 1968). Therefore, for engineering purposes, a simple method that is able to accurately predict the stage-discharge curve is worth investigating.

In this research, three groups of experiments were conducted in a large-scale meandering compound channel (Figure 1). The detailed 3D velocities and Reynolds shear stresses were recorded along a meander. Based on these experimental data, the appropriate cross-section for predicting discharges is determined after the flow patterns are analysed in the meandering main channel. A governing equation in curvilinear coordinates is derived from the momentum equation and the flow continuity equation. In this equation, two shear stress terms are reasonably ignored due to their negligible

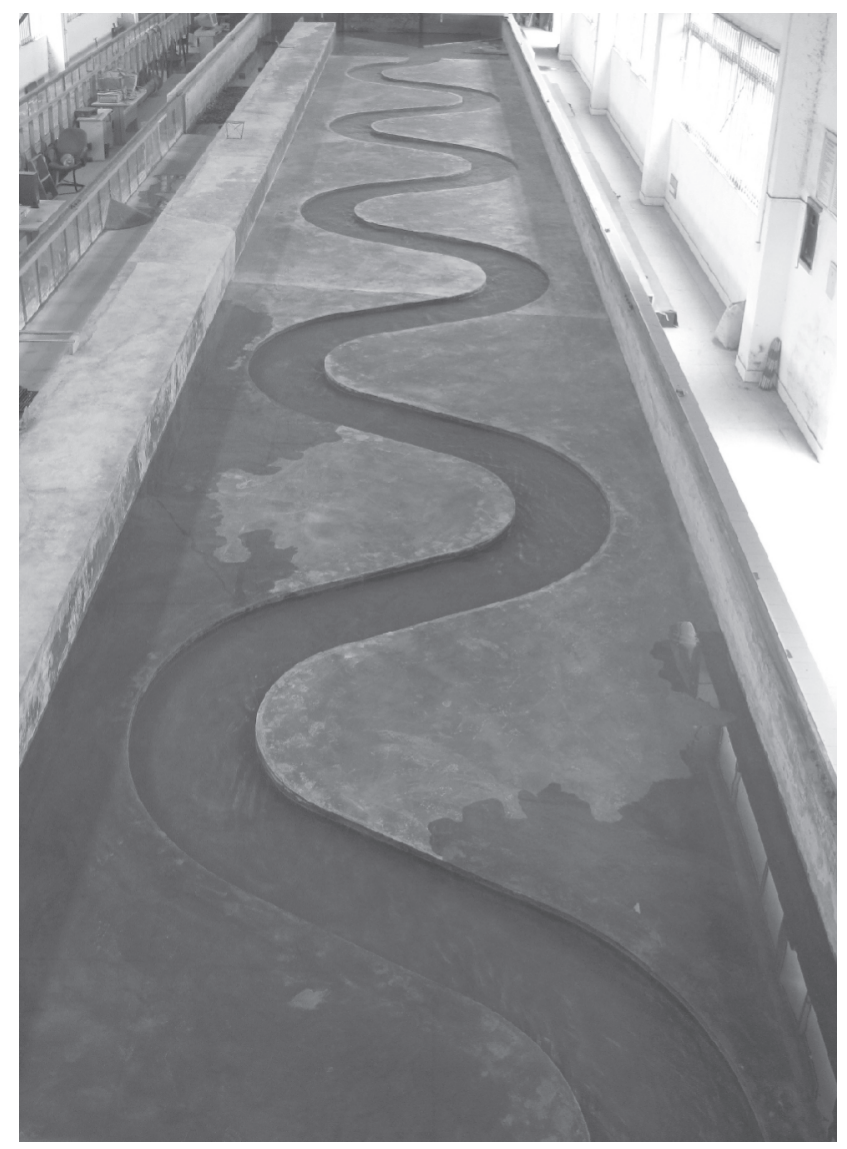

Figure 1. Photograph of the large-scale meandering compound channel at SKLH (looking downstream). This channel is a $35 \mathrm{~m}$-long, $4 \mathrm{~m}$-wide and $1 \mathrm{~m}$-high experimental flume

contributions. Thus, simple analytical solutions are obtained in the main channel and on the floodplain. Finally, the proposed model is verified using several groups of experimental data from the published literature.

\section{Experimental data used}

\subsection{New experiments}

The present experiments were conducted in a large-scale meandering compound channel constructed in a $35 \mathrm{~m}$-long, $4 \mathrm{~m}$ wide and $1 \mathrm{~m}$-high flume at the State Key Laboratory of Hydraulics and Mountain River Engineering (SKLH) at Sichuan University in China (Figure 1). In this meandering channel, the stage-discharge curve was measured using an automatic ultrasonic measurement system produced by the Sinfotek Corporation. The 3D velocities, turbulences and Reynolds shear stresses were recorded by a three-component Sontek acoustic Doppler velocimeter for three cases (i.e. MN1, MN2 and MN3) with relative flow depths of $D_{\mathrm{r}} \approx 0 \cdot 25,0 \cdot 35$ and $0 \cdot 45$, respectively, where $D_{\mathrm{r}}=(H-h) / H, H$ is the flow depth and $h$ is the bankfull height (Figure 2). The original point of the curvilinear coordinate system was set at the right-side corner of the main channel at CS1. The shape of the main channel was rectangular 
Water Management

Volume 169 Issue WM1
Method for assessing discharge in

meandering compound channels

Liu, Shan, Liu and Yang

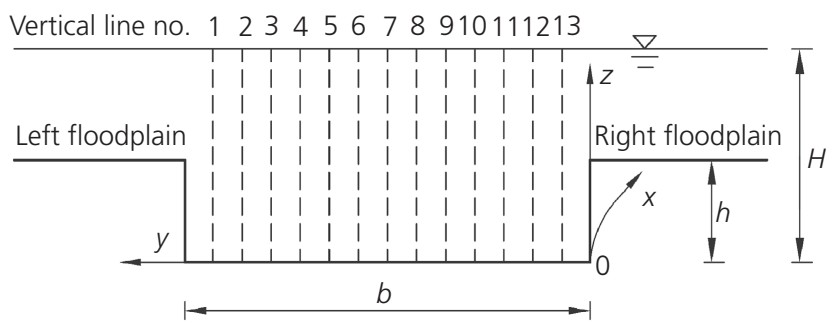

Figure 2. Cross-section of the meandering main channel with 13 vertical measurement lines at CS1. In the experiments, the channel width $(b)$ and the bankfull depth $(h)$ are 0.7 and $0.14 \mathrm{~m}$, respectively. The lateral interval between vertical measurement lines is $0.05 \mathrm{~m}$

with a sinuosity $s=1 \cdot 381$. The valley slope $\left(S_{\mathrm{fp}}\right)$ was $0 \cdot 001$. In the meandering main channel, the lateral interval of 13 vertical measurement lines was $0.05 \mathrm{~m}$. The measurement intervals between two vertical points were arranged as $0.015 \mathrm{~m}$.

Four and a half meanders were constructed in this large flume. The top view of the test meander is shown in Figure 3. The width of the main channel $(b)$ was designed as $0.7 \mathrm{~m}$, giving an aspect ratio $(b / h)$ of $5 \cdot 0$. The inner radius $(r)$ and the outer radius $(R)$ were 0.9 and $1.6 \mathrm{~m}$, respectively, such that the medium radius $\left(R_{\mathrm{m}}\right)$ was $1.25 \mathrm{~m}$ The details of the geometry parameters are shown in Table 1. All experiments were conducted under quasi-uniform flow conditions by ensuring that the water surface slope remained parallel to the bed slope at each meander. This condition was achieved by manually adjusting the tailgate at the end of the flume. When the deviation between the water surface and the bed slope reached less than $5 \%$, it was determined that quasi-uniform flow had been attained, and then measurements commenced.

\subsection{Data from the published literature}

Data were selected from the published literature (Lambert and Sellin, 1996; Shiono et al., 1999; Spooner, 2001), and details of the experimental parameters are shown in Table 2. Different scales and geometry parameters might have different effects on the predictive accuracy, and, therefore, these data were selected from large and small meandering channels with different sinuosities. Brief introductions for these experiments are provided for convenience.

\subsubsection{Source 1}

The experimental flume consisted of a rectangular crosssection that was $13 \mathrm{~m}$ long, $2.4 \mathrm{~m}$ wide and $0.3 \mathrm{~m}$ deep with a valley slope $S_{\mathrm{fp}}=0.002$ located at Loughborough University. This flume was constructed from grass and acrylic. The width of the main channel was $0.4 \mathrm{~m}$, with a bankfull level of $0.04 \mathrm{~m}$, giving an aspect ratio of $h / b=10$. The data acquisition system and experimental set-up are described in detail by Spooner (2001)

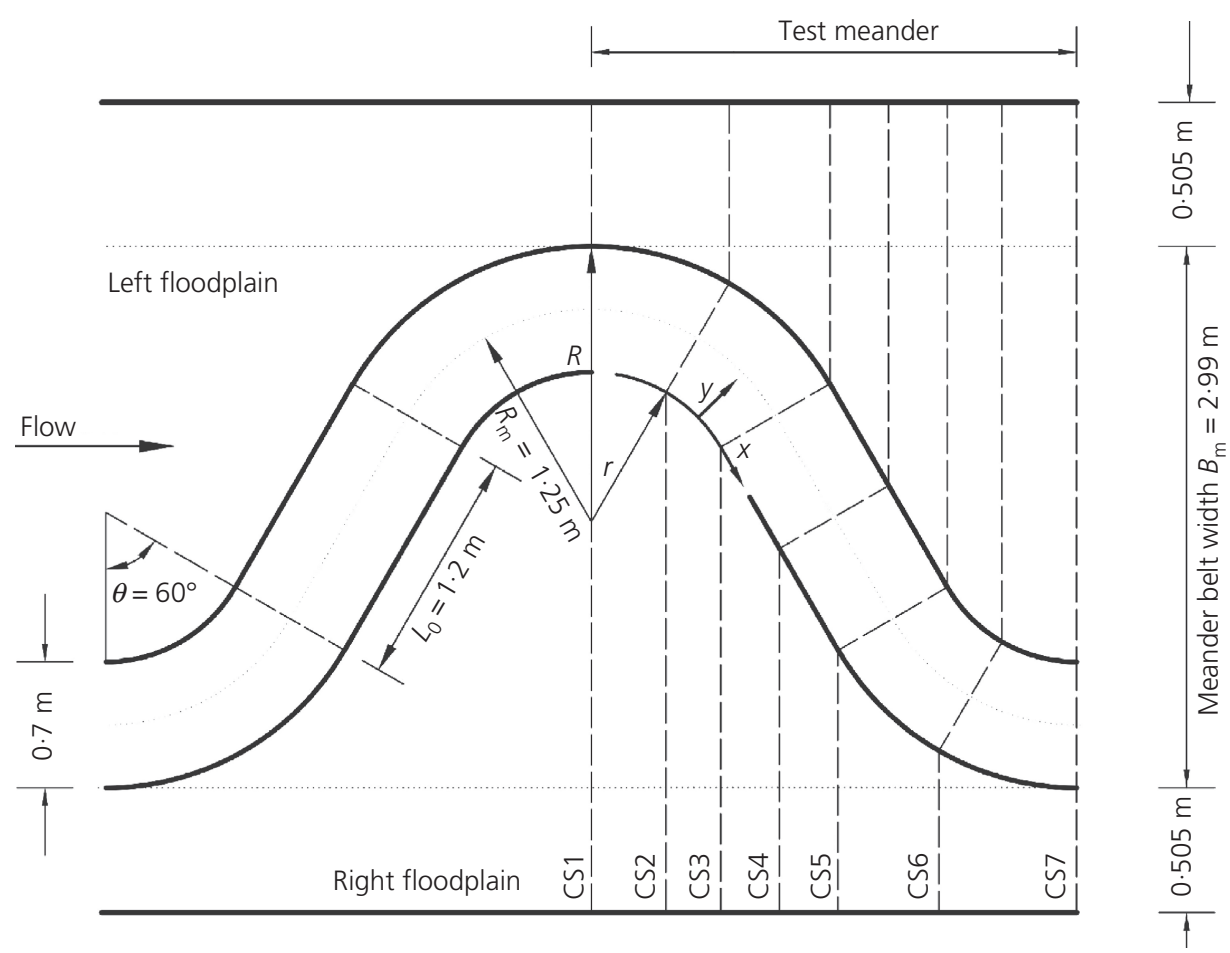

Figure 3. Top view of the test meander with the curvilinear

co-ordinate system 


\begin{tabular}{|c|c|c|c|c|c|c|c|c|}
\hline $\begin{array}{l}\text { Total } \\
\text { channel } \\
\text { width, B: m }\end{array}$ & $\begin{array}{c}\text { Meander } \\
\text { belt } \\
\text { width, } B_{\mathrm{m}}: \mathrm{m}\end{array}$ & $\begin{array}{c}\text { Wavelength: } \\
\text { m }\end{array}$ & $\begin{array}{l}\text { Inner } \\
\text { radius, } \\
r: \mathrm{m}\end{array}$ & $\begin{array}{l}\text { Outer } \\
\text { radius, } \\
R: \mathrm{m}\end{array}$ & $\begin{array}{c}\text { Cross-over } \\
\text { length, } \\
L_{0}: m\end{array}$ & $\begin{array}{c}\text { Side } \\
\text { slope: } \\
\text { degrees }\end{array}$ & $\begin{array}{c}\text { Valley } \\
\text { slope, } S_{f p}\end{array}$ & $\begin{array}{c}\text { Sinuosity, } \\
\text { s }\end{array}$ \\
\hline $4 \cdot 0$ & $2 \cdot 99$ & $5 \cdot 53$ & 0.9 & $1 \cdot 6$ & $1 \cdot 2$ & 90 & 0.001 & $1 \cdot 381$ \\
\hline Series name & $\begin{array}{c}\text { Discharge, } \\
\text { Q: } \mathrm{m}^{3} / \mathrm{s}\end{array}$ & \multicolumn{2}{|c|}{$\begin{array}{c}\text { Flow depth, } \\
H: \mathrm{m}\end{array}$} & $\begin{array}{c}\text { Bankfull } \\
\text { depth, } h: \mathrm{m}\end{array}$ & \multicolumn{2}{|c|}{$\begin{array}{c}\text { Relative flow } \\
\text { depth, } D_{\mathrm{r}}\end{array}$} & \multicolumn{2}{|c|}{ Floodplain condition } \\
\hline MN1 & $0 \cdot 189$ & \multicolumn{2}{|c|}{$0 \cdot 255$} & \multicolumn{2}{|c|}{$0 \cdot 14$} & \multicolumn{2}{|c|}{0.451} & Smooth \\
\hline MN2 & $0 \cdot 113$ & \multicolumn{2}{|c|}{0.216} & \multicolumn{2}{|c|}{$0 \cdot 14$} & \multicolumn{2}{|c|}{0.352} & Smooth \\
\hline MN3 & 0.085 & \multicolumn{2}{|c|}{0.189} & \multicolumn{2}{|c|}{$0 \cdot 14$} & \multicolumn{2}{|c|}{0.259} & Smooth \\
\hline
\end{tabular}

Table 1. Geometry parameters and experimental conditions

\begin{tabular}{|c|c|c|c|c|c|c|c|c|}
\hline Source & $\begin{array}{l}\text { Valley slope, } \\
\qquad S_{f p}\end{array}$ & $\begin{array}{c}\text { Main channel, } \\
S_{\mathrm{mc}}\end{array}$ & $\begin{array}{c}\text { Sinuosity, } \\
\text { s }\end{array}$ & $\begin{array}{c}\text { Main } \\
\text { channel } \\
\text { width, b: m }\end{array}$ & $\begin{array}{l}\text { Channel } \\
\text { width, } \\
\text { B: m }\end{array}$ & $\begin{array}{c}\text { Bankfull } \\
\text { depth, } \\
\text { h: m }\end{array}$ & $\begin{array}{l}\text { Medium } \\
\text { radius, } \\
R_{\mathrm{m}}: \mathrm{m}\end{array}$ & $\begin{array}{c}\text { Cross-over } \\
\text { length, } \\
L_{0}: \mathrm{m}\end{array}$ \\
\hline Spooner (2001) & 0.002 & 0.001445 & $1 \cdot 384$ & $0 \cdot 4$ & $2 \cdot 4$ & 0.04 & 0.765 & 0.75 \\
\hline $\begin{array}{c}\text { Case 2, Shiono } \\
\text { et al. (1999) }\end{array}$ & $0.001-0.002$ & $0.00073-0.00146$ & $1 \cdot 372$ & $0 \cdot 152$ & $1 \cdot 2$ & 0.052 & $0 \cdot 425$ & $0 \cdot 376$ \\
\hline $\begin{array}{c}\text { Case 4, Shiono } \\
\text { et al. (1999) }\end{array}$ & $0.0005-0.002$ & $0 \cdot 000458-0.00183$ & $1 \cdot 092$ & $0 \cdot 15$ & $1 \cdot 2$ & 0.052 & $0 \cdot 425$ & $0 \cdot 377$ \\
\hline $\begin{array}{c}\text { Case 5, Shiono } \\
\text { et al. (1999) }\end{array}$ & $0.0005-0.002$ & $0.000318-0.00127$ & $1 \cdot 571$ & $0 \cdot 15$ & $1 \cdot 2$ & 0.052 & $0 \cdot 425$ & 0 \\
\hline $\begin{array}{l}\text { Lambert and } \\
\text { Sellin (1996) }\end{array}$ & 0.001 & 0.000847 & $1 \cdot 18$ & $0 \cdot 85$ & $4 \cdot 9$ & $0 \cdot 15$ & $1 \cdot 365$ & 0 \\
\hline
\end{tabular}

\subsubsection{Source 2}

A series of experiments was conducted in a $9 \cdot 2 \mathrm{~m}$-long, $1.2 \mathrm{~m}$ wide and $0.35 \mathrm{~m}$-high flume in the hydraulics laboratory at the University of Bradford (Shiono et al., 1999). The entire flume was made of acrylic, with different meandering channel configurations. Five cases (1-5) were investigated in different meandering channels. The data measured in the channel with small $(s=1 \cdot 092$, case 4$)$, medium $(s=1 \cdot 372$, case 2$)$ and large sinuosities $(s=1 \cdot 571$, case 5$)$ were selected. Different valley slopes $\left(S_{\mathrm{fp}}=0 \cdot 0005-0 \cdot 002\right)$ were also considered. In these three cases, the detailed measurements of the velocities were collected by way of laser Doppler anemometry under two relative flow depths of $D_{\mathrm{r}}=0 \cdot 15$ and $0 \cdot 5$ (Shiono and Muto, 1998). These velocity data were provided by Professor Koji Shiono at Loughborough University and Professor Muto Yasunori at the University of Tokushima. Hence, further discussion of the velocity parameter is possible.

\subsubsection{Source 3}

A distorted 1:5 scale model was constructed in the $56 \mathrm{~m}$-long Flood Channel Facility at the laboratory of HR Wallingford in the UK. This model was moulded in cement mortar with a valley slope $S_{\mathrm{fp}}=0.001$. The main channel was $0.85 \mathrm{~m}$ wide and $0.15 \mathrm{~m}$ deep, giving an aspect ratio $(b / h)$ of 5.67 , and the sinuosity was $1 \cdot 18$. Once the stage-discharge relationship was obtained, detailed velocity measurements were conducted for three typical flow depths. Hence, sub-area discharges were obtained. Additional details can be found in Lambert and Sellin (1996).

\section{Flow patterns at the apex and the middle cross-section}

Before introducing the theoretical background, the flow patterns at different cross-sections in the meandering compound channel must be understood. Based on the experimental data, the streamwise velocity distribution is presented at two typical cross-sections (the apex section CS1 and the middle crosssection CS4), as shown in Figure 4. From this figure, it can be observed that the magnitude of the velocity at CS1 appears larger than that at CS4. At the apex, dense contour lines are noted at the upper region next to the left-hand floodplain; however, dense contour lines are observed at the bankfull level near the right-side floodplain (black dashed circles in Figure 4) 
Method for assessing discharge in meandering compound channels Liu, Shan, Liu and Yang

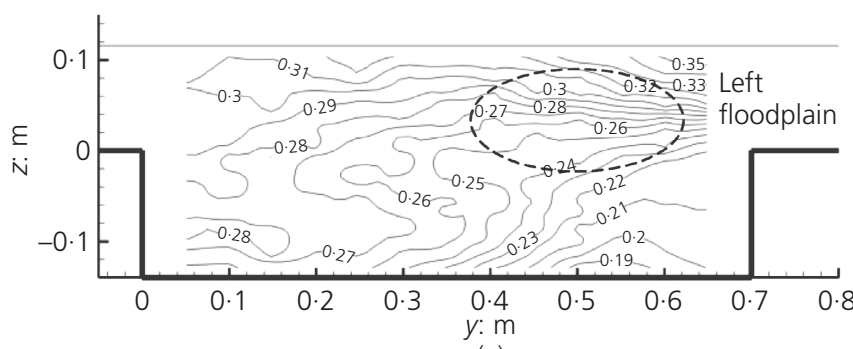

(a)

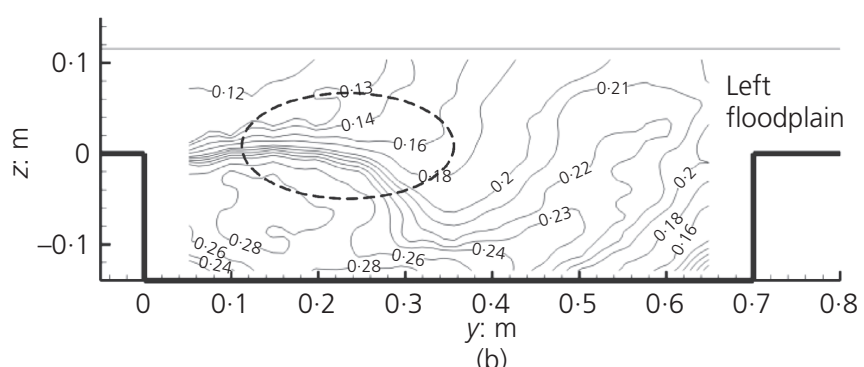

(b)

Figure 4. Streamwise velocity $(U)$ distributions in the meandering main channel at two typical sections (the black dashed circle indicates the high vertical gradient of the $U$ zone): (a) apex section (CS1), (b) middle cross-section (CS4)

in CS4 because the upstream flood plain flow plunges into the meandering main channel and collides with the main channel flow. This process converts additional kinetic energy into heat by producing much stronger vertical shear stresses (Figure 5) and local super elevation. Therefore, the conveyance capability at the middle cross-section is weaker than that at the apex. In addition, Liu et al. (2014a) and Shiono and Muto (1998) have observed that the flow directions in the main channel and floodplain are the same at the apex section (CS1) and different at the middle cross-section (CS4). In summary, the flow pattern is uniform at the apex section, where the flow directions in the main channel and on the floodplain are the same. Therefore, it is suggested that the apex section is the best crosssection in the meandering compound channel for prediction of the stage-discharge curve.

\section{Theoretical background}

It is more suitable to use the momentum equation and the flow continuity equation in curvilinear co-ordinates in the meandering channel. Spooner and Shiono (2003) used 2D curvilinear equations, and developed a method for modelling the depth-averaged velocity in meandering compound channels.

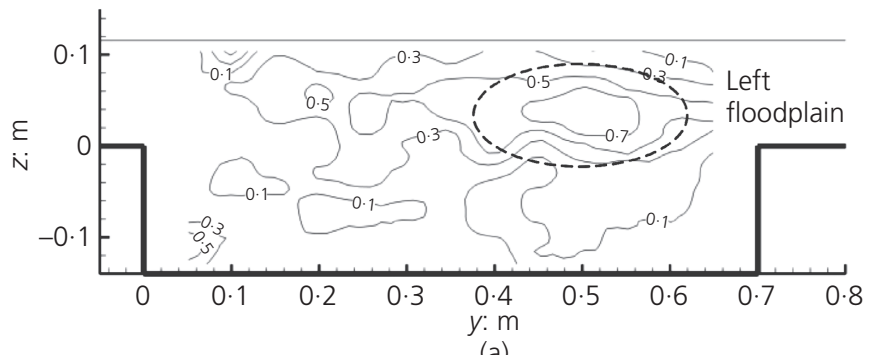

(a)

Figure 5. Vertical shear stress $\left(\tau_{z x}\right)$ distributions in the meandering main channel at two typical sections (the black dashed circle indicates the strong $\tau_{z x}$ zone): (a) apex section (CS1); (b) middle cross-section (CS4)
Under the curvilinear co-ordinate system, the $x$ axis is parallel to the meandering main channel, and the $y$ axis is normal to the meandering main channel (see Figure 3). For the condition of quasi-uniform flow in the meandering channel, the streamwise momentum equation and the flow continuity equation in curvilinear co-ordinates are combined, and the result is

$$
\begin{aligned}
\frac{r}{r+y} & \frac{\partial \rho U^{2}}{\partial x}+\frac{\partial \rho U V}{\partial y}+\frac{\partial \rho U W}{\partial z}+\frac{2 \rho U V}{r+y} \\
= & \frac{r}{r+y} \rho g S+\frac{\partial \tau_{x x}}{\partial x}+\frac{\partial \tau_{y x}}{\partial y}+\frac{\partial \tau_{z x}}{\partial z}+\frac{2 \tau_{y x}}{r+y}
\end{aligned}
$$

where $x, y$ and $z$ are the streamwise, lateral and vertical directions, respectively; $U, V$ and $W$ are the velocity components corresponding to the $x, y$ and $z$ directions, respectively; $\rho$ is the flow density; $g$ is the local gravitational acceleration; $S$ is the bed slope; and $\tau_{x x}, \tau_{y x}$ and $\tau_{z x}$ are the Reynolds shear stresses.

In Equation 1, $\partial \rho U V / \partial y$ and $2 \rho U V / r+y$ represent the same dimension, and thus it is assumed that $2 \rho U V /(r+y)=\phi(\partial \rho U V /$ $\partial y$ ), where $\phi$ is a dimensionless coefficient. $\phi=0$ when $r \rightarrow \infty$.

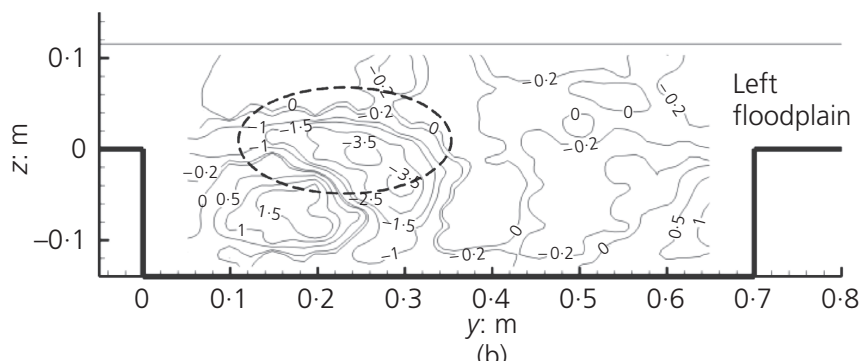


According to the generation mechanism of secondary flows in the meandering channel (Liu et al., 2014a), in the main channel the secondary currents consist of the original secondary current cell $\left(V_{\mathrm{o}}\right)$ enhanced by the centrifugal force $\left(F_{\mathrm{c}}\right)$ and the component of floodplain flow $\left(V_{\mathrm{fp}}\right)$. Liu et al. (2014a) suggested $F_{\mathrm{c}}=k\left[\partial\left(\rho U V_{\mathrm{o}}\right) / \partial y\right]$, where $k$ is a dimensionless coefficient reflecting the relationship between secondary flows and $F_{\mathrm{c}}$. Hence, $V=(1+k) V_{\mathrm{o}}+V_{\mathrm{fp}}$. Thus, Equation 1 may be rewritten as

$$
\text { 2. } \begin{aligned}
\frac{\partial \rho U^{2}}{\partial x}+ & \frac{r+y}{r}(1+\phi)\left[(1+k) \frac{\partial \rho U V_{\mathrm{o}}}{\partial y}+\frac{\partial \rho U V_{f p}}{\partial y}\right] \\
& +\frac{r+y}{r} \frac{\partial \rho U W}{\partial z} \\
= & \rho g S+\frac{r+y}{r}\left(\frac{\partial \tau_{x x}}{\partial x}+\frac{\partial \tau_{y x}}{\partial y}+\frac{\partial \tau_{z x}}{\partial z}\right)+\frac{2 \tau_{y x}}{r}
\end{aligned}
$$

In this research, the focus is on the sub-area discharge and the total discharge. Darby and Thorne (1996) and Liu et al. (2014b) proposed simplified versions of the flow momentum and continuity equations for the lateral distribution of the local unit discharge $\left(q=\int_{0}^{H} U \mathrm{~d} z\right)$ in a vegetated straight compound channel. As such, Equation 2 is integrated over the flow depth, and Equation 3 is obtained as

3.

$$
\begin{aligned}
\underbrace{\Gamma}_{1}= & \underbrace{\rho g H S}_{2}+\underbrace{\frac{\partial}{\partial y}\left(\frac{1}{2} \rho \frac{r+y}{r} \lambda \sqrt{\frac{f}{8}} \frac{\partial q^{2}}{\partial y}\right)}_{3} \\
& -\underbrace{\frac{\rho}{H^{2}} \frac{r+y}{r} \frac{f}{8} q^{2}}_{4}+\underbrace{\frac{2}{r}\left(\frac{1}{2} \rho \lambda \sqrt{\frac{f}{8}} \frac{\partial q^{2}}{\partial y}\right)}_{5}
\end{aligned}
$$

4.

$$
\begin{aligned}
\overline{\tau_{y x}} & =\frac{\rho \varepsilon}{H} \frac{\partial q}{\partial y} & \tau_{b} & =\frac{\rho}{H^{2}} \frac{f}{8} q^{2} \\
\varepsilon & =\lambda H U_{*} & U_{*} & =\frac{1}{H} \sqrt{\frac{f}{8}} q
\end{aligned}
$$

where $\Gamma$ is the velocity parameter that represents the longitudinal velocity variation and lateral secondary flow variation; $\lambda$ is the dimensionless eddy viscosity; $f$ is the Darcy-Weisbach friction factor; $\overline{\tau_{y x}}$ is the depth-averaged Reynolds shear stress; $\varepsilon$ is the depth-averaged eddy viscosity; and $U_{*}$ is the shear velocity. In Equation 3 'term 1' through 'term 5' represent the velocity variation term, the gravity term, the gradient of shear stress term, the bed shear stress term and the shear stress term, respectively.

At the apex section, the component of floodplain flow $\left(V_{\mathrm{fp}}\right)$ can be ignored because the angle between the streamwise direction and the flow direction is nearly $0^{\circ}$ (Liu et al., 2014a). Therefore, the velocity parameter is

$$
\text { 5. } \quad \Gamma=\frac{r}{r+y} \frac{\partial \rho H U_{\mathrm{d}}^{2}}{\partial x}+(1+\phi)\left[(1+k) \frac{\partial(\rho H U V o)_{\mathrm{d}}}{\partial y}\right]
$$

where $U_{\mathrm{d}}$ is the depth-averaged velocity.

Noting that the velocity term in Equation 5 is expressed in velocity components $(U$ and $V$ ), it is easy to understand its physical significance associated with the longitudinal velocity variation and the lateral secondary flow variation. Using the present study's experimental data (MN1), longitudinal profiles of $U_{\mathrm{d}}^{2}$ at seven vertical measurement lines (lines 1, 3, 5, 7, 9, 11 and 13) are shown in Figure 6. From this figure, it is noted that for these seven vertical lines the maximal $U_{\mathrm{d}}^{2}$ value always occurs at the apex sections. In addition, the mirror longitudinal profiles of $U_{\mathrm{d}}^{2}$ at lines 1 and 13 are observed because of the periodical flow pattern in the meandering channel. Therefore, $\partial U_{\mathrm{d}}^{2} / \partial x=0$ at the apexes due to the extreme-value theory. Thus, Equation 5 can be further simplified as

6. $\Gamma=(1+\phi)(1+k) \frac{\partial\left(\rho H U V_{\mathrm{o}}\right)_{\mathrm{d}}}{\partial y}=(1+\phi) \frac{\partial(\rho H U V)_{\mathrm{d}}}{\partial y}$

Equation 6 advances the understanding that, at the apex section, the secondary flow is enhanced only by the centrifugal force, and it demonstrates that good prediction at the apex section can still be obtained by ignoring the longitudinal velocity variation, similar to the work of Ervine et al. (2000), Huai et al. (2009) and McGahey and Samuels (2003).

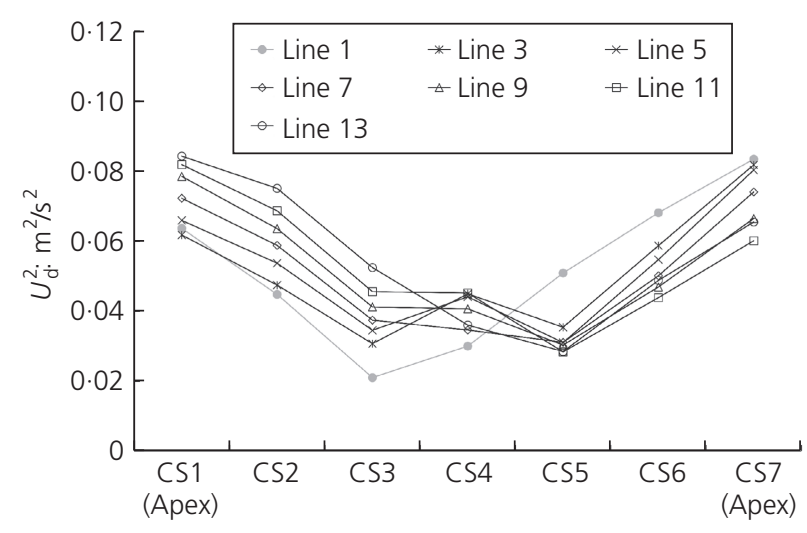

Figure 6. Longitudinal distributions of $U_{\mathrm{d}}^{2}$ at measurement lines 1 , $3,5,7,9,11$, and 13 (see Figure 3 for lateral locations) in the meandering main channel based on the experimental data (MN1) 
Water Management

Volume 169 Issue WM1
Method for assessing discharge in

meandering compound channels

Liu, Shan, Liu and Yang
It is practically impossible to solve Equation 3 analytically because it takes the form of an inhomogeneous differential equation when the parameters are given. This difficulty arises because the bed shear stress term (term 4) simultaneously contains the independent variable $(y)$ and the dependent $(q)$. Therefore, one or more terms must be ignored to obtain the analytical solution of Equation 3. To address this difficulty, the orders of magnitude of the five terms are compared. Several cases are selected from different sources with different channel scales, flow depths and geometry conditions (from the data from the experiments reported here and those of Shiono and Muto (1998) and Spooner (2001)). Most importantly, the subarea discharges are reported in these cases. To conveniently conduct comparisons, the contribution percentage $\left(C_{\mathrm{p}}\right)$ is defined as

7. $C_{\mathrm{p}}=\left|\operatorname{term} j / \sum_{j=1}^{5} \operatorname{term} j\right| \times 100 \%$

where $j=1-5$ in Equation 3. For different cases, the $C_{\mathrm{p}}$ values of five terms are examined, as shown in Figure 7. From this figure, the gravity term shows the highest proportion of contribution (approximately 50\%). The second largest contribution $(35.42 \%)$ is from the velocity term, and the third comes from the bed shear stress term (14.21\%). However, the shear stress term and its gradient term account for only $0.31 \%$ and $0.89 \%$,

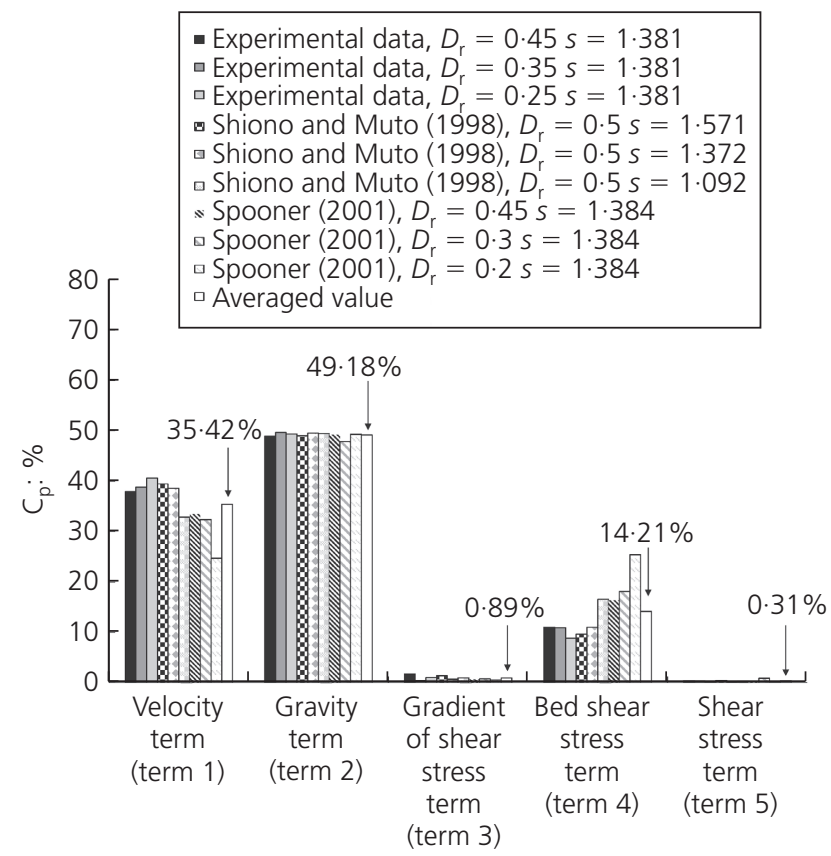

Figure 7. Comparison of the contribution percentage $\left(C_{p}\right)$ for five terms in Equation 3 based on the data from the experiments reported here and those of Shiono and Muto (1998) and Spooner (2001) respectively, which indicates that the two shear stress terms can be ignored due to their negligible contributions. Therefore, the simple relationship

8. $\Gamma=\rho g H S-\frac{\rho}{H^{2}} \frac{r+y f}{r} \frac{\gamma}{8} q^{2}$

is obtained. In Equation 8, the error induced by ignoring two shear stress terms is noted. In Equations 3 and 8, the gravity term and the bed shear stress term are the same, and hence, the velocity parameter $(\Gamma)$ reflects the prediction error. From this analysis, the $C_{\mathrm{p}}$ values of term 3 and term 5 in Equation 3 are only $0.89 \%$ and $0.31 \%$, respectively. Therefore, the difference between the velocity parameters in Equations 3 and 8 is only $1 \cdot 2 \%$. The impact of this difference on the prediction will be addressed in the discussion.

Equation 8 is similar to the simplified equation proposed by Spooner and Shiono (2003), but the largest difference between the two approaches is the definition of the velocity variation term (i.e. the left-hand side of Equation 8). Spooner and Shiono (2003) used a term that included a parameter $K$ and $U_{\mathrm{d}}^{2}$ to replace the velocity variation term. However, in this paper, the velocity variation term is assumed to be a constant across the channel. To verify this assumption, the velocity parameter is back-calculated using the experimental data (MN1, MN2 and MN3), as shown in Figure 8. From this figure, the lateral profiles of $\Gamma$ indicate that this assumption is reasonable but is only demonstrated at $D_{\mathrm{r}} \geq 0 \cdot 25$. Spooner and Shiono (2003) observed large lateral variations of the velocity variation term in low-discharge cases $\left(D_{\mathrm{r}}=0 \cdot 0\right.$ and $\left.0 \cdot 2\right)$. This point will be discussed further with respect to applications.

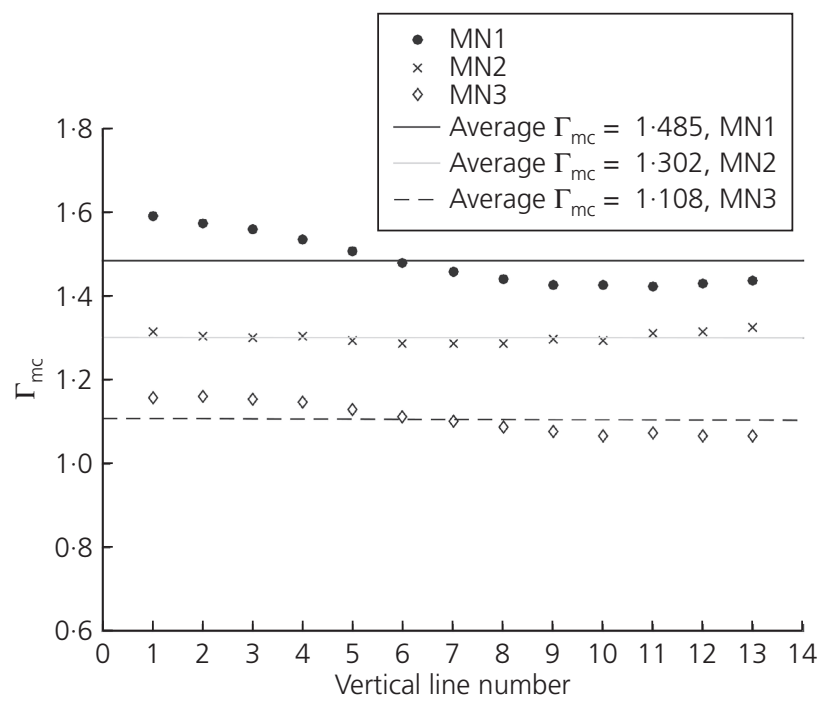

Figure 8. Lateral profiles of the velocity parameter $\Gamma_{j}$ in the meandering main channel based on the experimental data (MN1, MN2 and MN3) 
Next, the analytical solution of the discharge per unit width in the meandering main channel is presented as

9. $q=\sqrt{\frac{H^{2}}{\rho} \frac{r}{r+y} \frac{8}{f}(\rho g H S-\Gamma)}$

Laterally integrating Equation 9, the main channel discharge $\left(Q_{\mathrm{mc}}\right)$ is obtained as

10. $Q_{\mathrm{mc}}=2(\sqrt{r(r+b)}-r) \sqrt{\frac{H^{2}}{\rho} \frac{8}{f_{\mathrm{mc}}}\left(\rho g H S_{\mathrm{mc}}-\Gamma_{\mathrm{mc}}\right)}$

where $S_{\mathrm{mc}}$ is the slope of meandering main channel $\left(=S_{\mathrm{fp}} / s\right)$.

On the floodplain, $r \rightarrow \infty$. Therefore, the floodplain discharge $\left(Q_{\mathrm{fp}}\right)$ is

11. $Q_{\mathrm{fp}}=(B-b) \sqrt{\frac{(H-h)^{2}}{\rho} \frac{8}{f_{\mathrm{fp}}}\left(\rho g(H-h) S_{\mathrm{fp}}-\Gamma_{\mathrm{fp}}\right)}$

where $B$ is the width of the entire channel.

Finally, the total discharge $\left(Q=Q_{\mathrm{mc}}+Q_{\mathrm{fp}}\right)$ is obtained. It should be noted that Equations 10 and 11 contain only two undetermined parameters (i.e. the Darcy-Weisbach friction factor $f_{i}$ and the velocity parameter $\Gamma_{i}$ ) when the geometry parameters are given in a meandering channel, where $i$ is 'mc' or 'fp', indicating the value in the main channel or on the floodplain, respectively. Two simple relationships, Equations 10 and 11 , are thus helpful in predicting the sub-area discharge and the stage-discharge.

\section{Determinations of parameters}

\subsection{Darcy-Weisbach friction factor}

The Darcy-Weisbach friction factor $\left(f_{i}\right)$ is usually backcalculated by the depth-averaged velocities and boundary shear stresses (Rameshwaran and Shiono, 2007; Shiono and Knight, 1991). However, instruments were not available for measuring the boundary shear stresses in the experiments reported here, and the data for bed shear stress were not reported by Lambert and Sellin (1996), Shiono et al. (1999) or Spooner (2001). Moreover, bed shear stresses are not often collected, particularly in natural rivers. Therefore, Equation 12, proposed by Huai et al. (2008) and Knight et al. (2007), is used to predict $f_{i}$ in the main channel and on the floodplain.

12. $f_{i}=\frac{8 g n^{2}}{R_{\mathrm{h}}^{1 / 3}}$

where $R_{\mathrm{h}}$ is the hydraulic radius, and $n$ is Manning's coefficient.
From Equation 12, the friction factor is only associated with Manning's coefficient $(n)$ and the hydraulic radius $\left(R_{\mathrm{h}}\right)$. Therefore, $f_{i}$ is determined when the channel bed condition and the flow depth are given. Liu et al. (2014a) used Equation 12 to predict $f_{i}$ in the meandering compound channel, and obtained accurate lateral distributions of depth-averaged velocity along a meander, thus demonstrating the predictive validity of Equation 12 for $f_{i}$.

\subsection{Velocity parameter}

The only undetermined parameter is the velocity parameter $\left(\Gamma_{i}\right)$. Although the expression for $\Gamma_{i}$ (see Equation 6) at the apex sections was obtained, its determination is still not straightforward. Shiono and Knight (1991) noted that the secondary flow term was not easily obtained by direct measurement, and backcalculation might be the best way to obtain its value. To predict discharges $(Q)$ at other flow depths, the sub-area discharges $\left(Q_{\mathrm{mc}}\right.$ and $\left.Q_{\mathrm{fp}}\right)$ are first needed, to back-calculate the values of $\Gamma_{i}$ in the main channel (Equation 10) and on the floodplain (Equation 11), respectively. Next, the three-order function between $\Gamma_{i}$ and $D_{\mathrm{r}}$ can be built. To obtain good predictions, at least three groups of $\Gamma_{i}$ and $D_{\mathrm{r}}$ are required. Once this information is at hand, the proposed model can predict the stagedischarge relationship for that same geometry. Therefore, two methods for obtaining $Q_{\mathrm{mc}}$ and $Q_{\mathrm{fp}}$ are shown below.

Method 1. $Q_{\mathrm{mc}}$ and $Q_{\mathrm{fp}}$ are obtained from the detailed experimental data, which are often available in indoor laboratory experiments, such as those reported here and Spooner's experiments (Spooner, 2001). Next, the velocity parameters are obtained by back-calculation.

However, in most cases, only the total discharges and flow depths are provided, particularly in natural rivers. Therefore, another method is presented to obtain the sub-area discharges.

Method 2. A classical equation was proposed by Knight and Demetriou (1983) to predict the main channel discharge, as follows.

13. $\frac{Q_{\mathrm{mc}}}{Q} \%=100\left(\frac{A_{\mathrm{mc}}}{A}+A_{\text {difference }}\right)$

where $Q_{\mathrm{mc}} / Q \%$ is the percentage of the flow carried by the main channel and $A_{\mathrm{mc}} / A$ is the ratio of the main channel flow area to the total flow area, which can be expressed as $1 /[(\alpha-1)$ $\left.D_{\mathrm{r}}+1\right]$ with $\alpha=B / b$, and $A_{\text {difference }}$ is a difference factor. Patra and Kar (2000) investigated $A_{\text {difference, and proposed the }}$ empirical expression

$$
\text { 14. } A_{\text {difference }}=108 \frac{1+36 D_{\mathrm{r}} \ln (s)}{\delta}\left(\frac{\alpha-1}{\alpha}\right)^{1 / 4}
$$

$$
\times\left(3 \cdot 3 D_{\mathrm{r}}\right)^{4 / a} \mathrm{e}^{-9 \cdot 9 D_{\mathrm{r}}}
$$


Water Management

Volume 169 Issue WM1
Method for assessing discharge in

meandering compound channels

Liu, Shan, Liu and Yang where $\delta$ is the ratio between the main channel width and its depth $(b / h)$. This equation reduces to the form of Knight and Demetriou (1983) in the straight channel when the sinuosity $(s)$ is $1 \cdot 0$. After the main channel discharge $\left(Q_{\mathrm{mc}}\right)$ is obtained, $Q_{\mathrm{fp}}\left(=Q-Q_{\mathrm{mc}}\right)$ is accordingly obtained. Finally, the velocity parameters are back-calculated in the main channel and on the floodplain.

\section{Application and discussion}

\subsection{Application in different cases}

Different sources of experimental data are used to verify the proposed model. Based on the detailed sub-area discharges from the experiments reported here and Spooner's experiments (Spooner, 2001), method 1 determines the velocity parameters, and Figure 9 shows that the predictive stage-discharge curves closely follow the experimental data. However, the data for the sub-area discharges are not sufficient (less than three groups) in the experiments of Lambert and Sellin (1996) and Shiono et al. (1999). Therefore, the velocity parameters are determined by method 2 in those cases. Figure 10 indicates that predictive discharges are approximately equal to the experimental data, although the subarea discharges are initially unknown. However, it should be noted that, in case 2, large discrepancies were observed at low discharges, which may be attributed to the assumption of the velocity parameter, which is considered to be a constant across the apex section. Spooner and Shiono (2003) reported that the velocity variation term varied significantly in the lateral direction in low-discharge cases $\left(D_{\mathrm{r}}=0 \cdot 0\right.$ and $\left.0 \cdot 2\right)$. Therefore, the assumption in this paper requires further investigation when the discharge is low. Nevertheless, in most cases, Figures 9 and 10 demonstrate that this model is capable of predicting the stage-discharge curve in meandering compound channels, whether method 1 or 2 is used to determine the velocity parameter.

\subsection{Discussion}

As previously mentioned in the foregoing section, the difference between the velocity parameters in Equations 3 and 8 is only $1 \cdot 2 \%$. To show the impact of this difference on the predictive discharge, the absolute relative error $\varepsilon_{\mathrm{r}}$ is proposed to avoid the offsetting of positive and negative values.

15. $\varepsilon_{\mathrm{r}}=\frac{1}{N} \sum_{1}^{N}\left|\frac{Q_{\text {predicted }}-Q_{\text {measured }}}{Q_{\text {measured }}}\right| \times 100 \%$

where $Q_{\text {predicted }}$ and $Q_{\text {measured }}$ are the predicted and measured discharges, respectively, and $N$ is the number of $Q_{\text {measured }}$. In this formulation, $Q$ is the total discharge $\left(=Q_{\mathrm{mc}}+Q_{\mathrm{fp}}\right)$.

The $\Gamma_{i}$ value is calculated using method 1 or 2, and Equation 3 and used to predict the discharges. The values of $\varepsilon_{\mathrm{r}}$ for all cases are shown in Table 3. From this table, the $\varepsilon_{\mathrm{r}}$ values from method 1 or 2 are within $5 \%$, indicating that the predictive capability of this model is good. The small difference

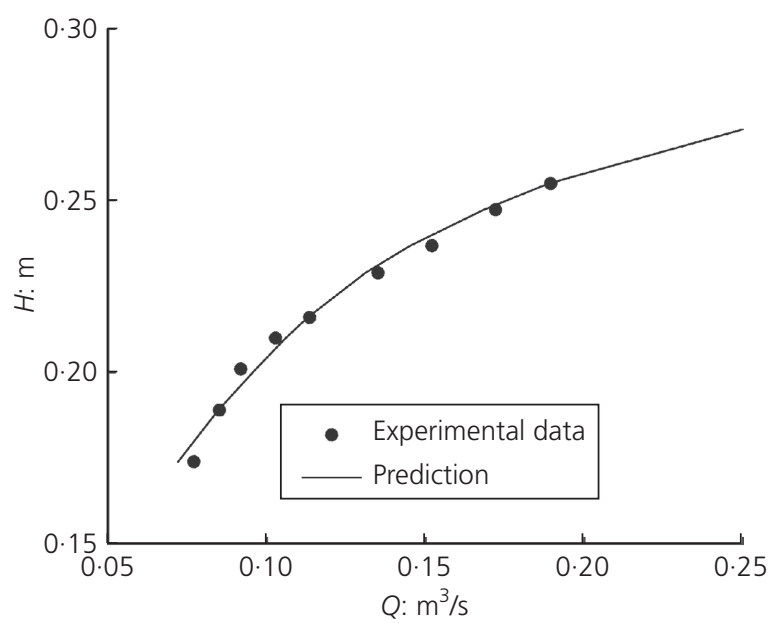

(a)

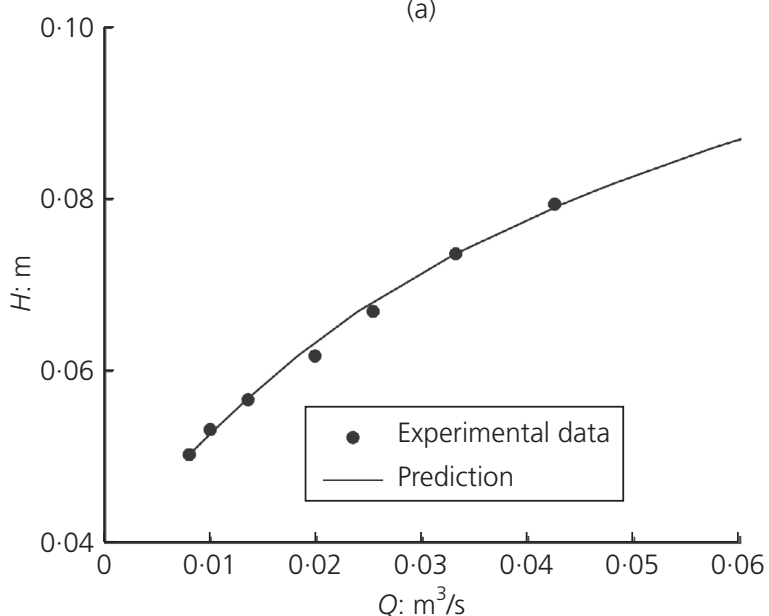

(b)

Figure 9. Comparison of the experimental data and the predictive stage-discharge curve with $\Gamma_{i}$ calculated by method 1 (see Section 5.2): (a) data from the experiments reported here; (b) data from Spooner (2001)

$(0 \cdot 13-2 \cdot 98 \%)$ between the values of $\varepsilon_{\mathrm{r}}^{\mathrm{a}}\left(\Gamma_{i}\right.$ determined by method 1 or 2$)$ and $\varepsilon_{\mathrm{r}}^{\mathrm{b}}\left(\Gamma_{i}\right.$ determined by Equation 3$)$ is also noted. The average difference of $\varepsilon_{\mathrm{r}}^{\mathrm{b}}-\varepsilon_{\mathrm{r}}^{\mathrm{a}}$ is only $1.55 \%$, thus demonstrating that it is reasonable to ignore the two shear stress terms in Equation 3. In addition, such a small error is acceptable for engineering purposes.

The sub-area discharges were reported in selected cases such that the vertical profiles of $\Gamma_{i}$ calculated by method 1 are shown in Figure 11. From this figure, $\Gamma_{i}$ is apparently a vertical variable that varies significantly in different meandering channels. In general, the $\Gamma_{\mathrm{mc}}$ values are larger than the $\Gamma_{\mathrm{fp}}$ values in the same meandering channel, which may be attributed to the centrifugal force in the main channel that enhances the secondary flow. The effect of the centrifugal force on the floodplain can be ignored such that $k=0$ in Equation 6. In addition, vertical co-ordinates may be more suitable on the floodplains, 


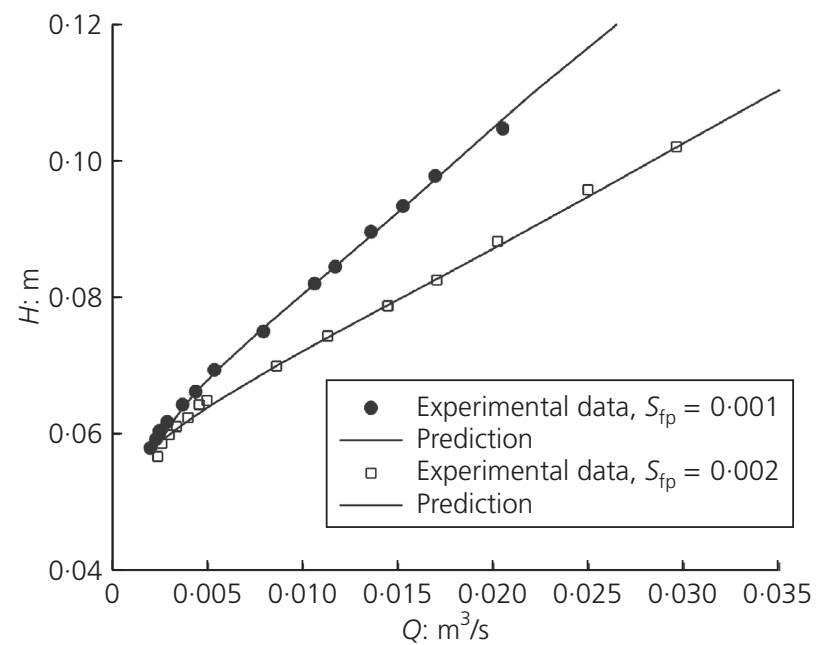

(a)

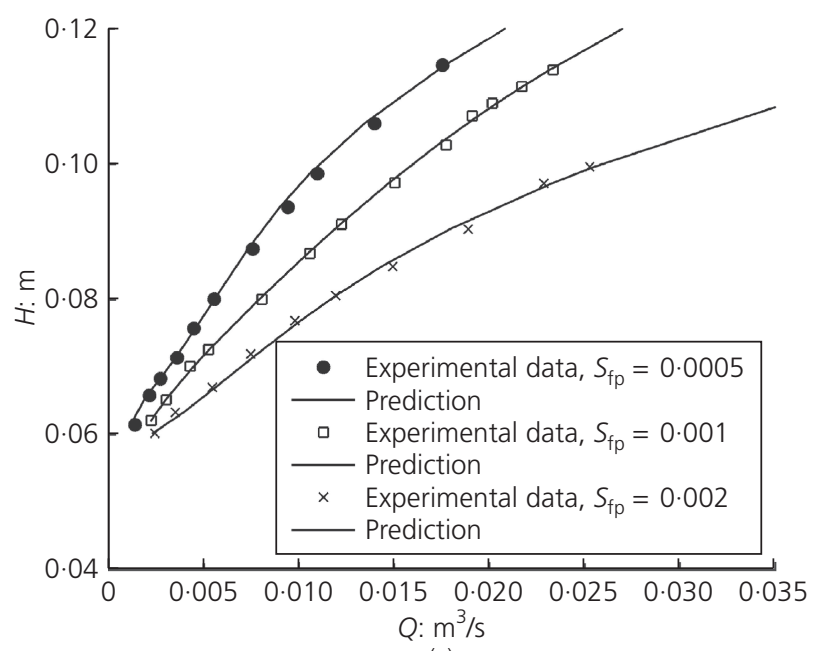

(c)

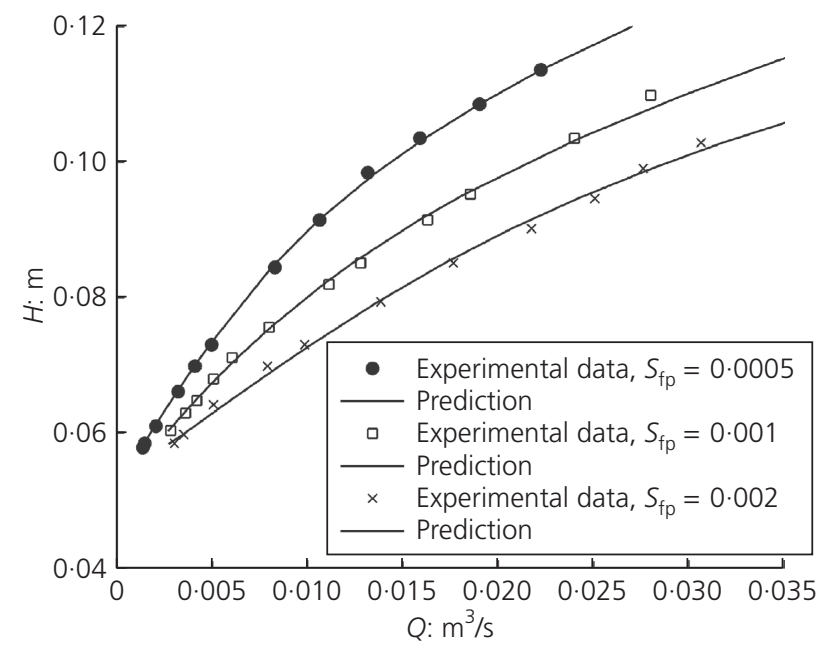

(b)

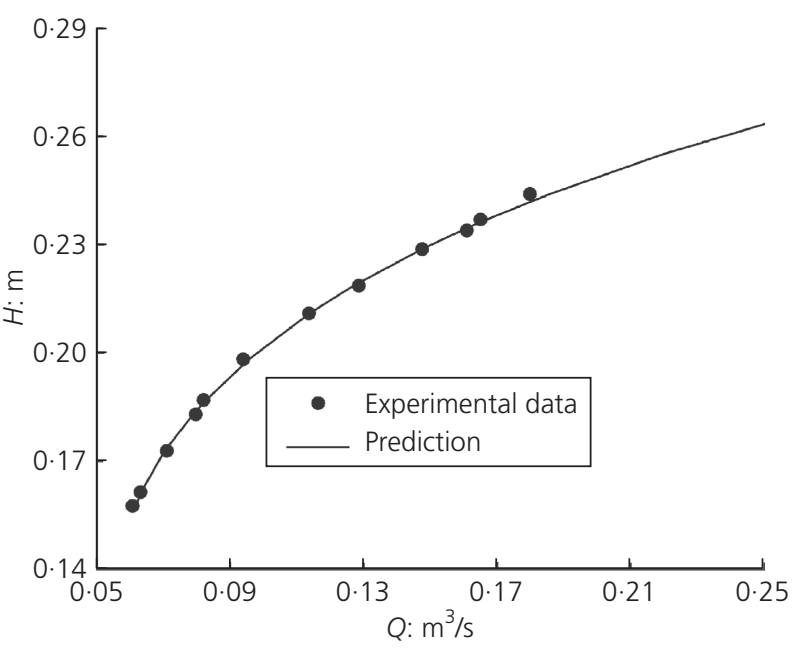

(d)

Figure 10. Comparison of the experimental data and the predictive stage-discharge curve with $\Gamma_{i}$ calculated by method 2 (see Section 5.2): (a) case 2, (b) case 4 and (c) case 5 from Shiono et al. (1999); (d) data from Lambert and Sellin (1996)

\begin{tabular}{|c|c|c|c|c|c|c|}
\hline & \multirow[b]{2}{*}{ Experimental data } & \multirow[b]{2}{*}{ Spooner (2001) } & \multicolumn{3}{|c|}{ Shiono et al. (1999) } & \multirow{2}{*}{$\begin{array}{l}\text { Lambert and Sellin } \\
\text { (1996) }\end{array}$} \\
\hline & & & Case 2 & Case 4 & Case 5 & \\
\hline$\varepsilon_{\mathrm{r}}^{\mathrm{a}}(\%)$ & $3 \cdot 45$ & $3 \cdot 05$ & $4 \cdot 24$ & $3 \cdot 11$ & $3 \cdot 25$ & $1 \cdot 15$ \\
\hline$\varepsilon_{\mathrm{r}}^{\mathrm{b}}(\%)$ & $4 \cdot 41$ & $5 \cdot 26$ & $4 \cdot 36$ & $4 \cdot 47$ & $6 \cdot 23$ & $2 \cdot 83$ \\
\hline$\varepsilon_{r}^{b}-\varepsilon_{r}^{a}(\%)$ & 0.96 & $2 \cdot 21$ & $0 \cdot 12$ & $1 \cdot 37$ & $2 \cdot 98$ & $1 \cdot 68$ \\
\hline
\end{tabular}

${ }^{\mathrm{a}} \Gamma_{i}$ calculated by method 1 or 2 in Section 5.2 .

${ }^{\mathrm{b}} \Gamma_{i}$ calculated by Equation 3.

Table 3. Absolute relative error $\left(\varepsilon_{\mathrm{r}}\right)$ for predictions using different velocity parameters 
Water Management

Volume 169 Issue WM1
Method for assessing discharge in

meandering compound channels

Liu, Shan, Liu and Yang and, thus, $r \rightarrow \infty$. Accordingly, $\phi=0$. Therefore, Equation 6 is simplified as $\Gamma=\partial\left(\rho H U V_{\mathrm{o}}\right)_{\mathrm{d}} / \partial y$, which is the expression of secondary flow on the floodplains.

An interesting phenomenon is shown in Figure 11. In the three channels with similar geometrical parameters (Shiono and Muto, 1998), the $\Gamma_{\mathrm{fp}}$ values are nearly identical, whereas the $\Gamma_{\mathrm{mc}}$ values vary with the sinuosities. These vertical distributions of $\Gamma_{i}$ indicate that the velocity parameter is determined by several factors (i.e. the channel scale, geometry and flow depth). For these reasons, an initial relationship for $\Gamma_{i}$ and $D_{\mathrm{r}}$ must be calibrated, following the steps introduced in Section 5.2 in a new meandering channel.

Indeed, Equation 9 is a modification of Manning's equation. If the secondary flow in the meandering channel is ignored (i.e. $\left.\Gamma_{i}=0\right)$ and the Darcy-Weisbach friction factor $\left(f_{i}\right)$ is substituted by Equation 12, Equation 9 may be rewritten as

16. $q=\frac{H}{n} \sqrt{\frac{r}{r+y} R^{1 / 3} H S}$

If the flow is located in a shallow and wide channel $(R \approx H)$, Equation 16 is changed into

17. $\frac{q}{H}=\sqrt{\frac{r}{r+y}} \frac{1}{n} H^{2 / 3} S^{1 / 2}$

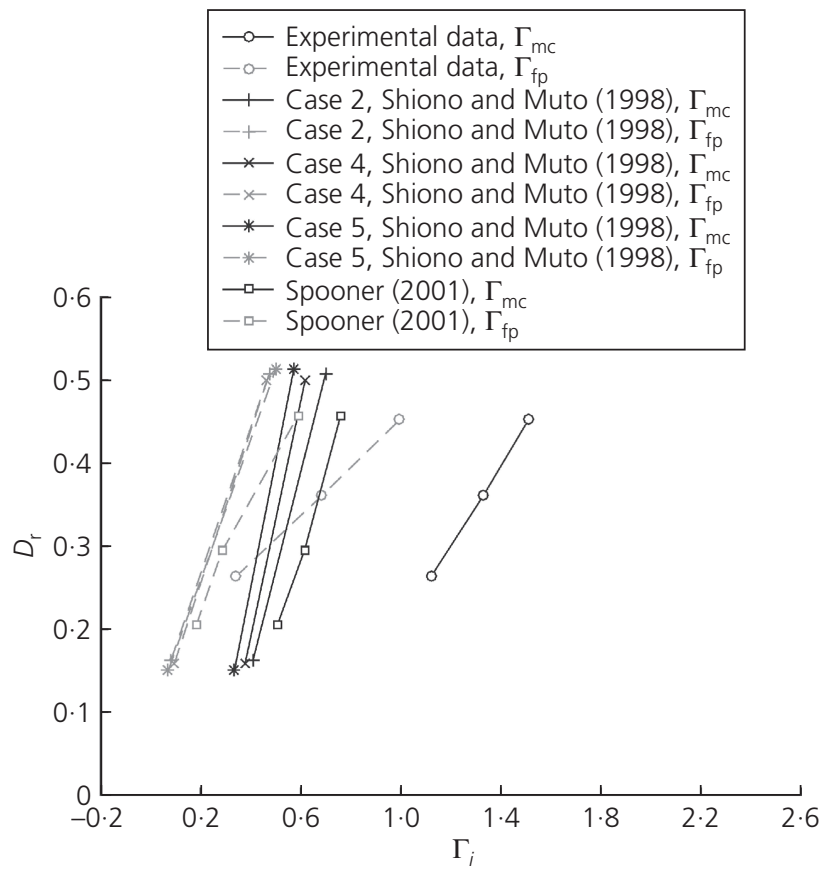

Figure 11. Vertical distributions of the velocity parameter in different cases based on the data from the experiments reported here and those of Shiono and Muto (1998) and Spooner (2001) which is Manning's equation in curvilinear co-ordinates. However, the classical Manning's equation is only a 1D method that cannot reflect the complicated 3D flow in the meandering channel. Therefore, poor results are obtained if Manning's equation is applied to a curved channel (Greenhill and Sellin, 1993). Although complex 3D flow occurs in the meandering channel, it is considered to be a $2 \mathrm{D}$ flow because it is reasonable to ignore the longitudinal velocity variation (see Section 4). This analysis explains why such good predictions are obtained in different meandering channels and also indicates that good predictions in the meandering channel must contain the effect of secondary flow.

The limitations of the proposed model are worth noting. In the process of derivation, the flow condition in the meandering channel is considered to be quasi-uniform flow. Therefore, further verification is needed under other flow conditions. In addition, initial calibration of the velocity parameter is important before the proposed model is used in a new channel. It is not easy to record the velocities and boundary shear stresses in a large range of discharges in rivers, but these data can be obtained in only three typical discharges. Next, the velocity parameter can be determined by method 1 or 2 , and accordingly the function between $\Gamma_{i}$ and $D_{\mathrm{r}}$ is obtained. Once these values are determined, the proposed model can predict the stage discharges in the same channel.

\section{Conclusion}

- Three groups of experiments were conducted in a large-scale meandering compound channel, and the detailed experimental data along a meander were recorded. Based on these data, the distributions of streamwise velocity and vertical shear stress at the apex and the middle cross-section were compared and analysed. The best position for predicting the stage-discharge curve is the apex section.

- The method for assessing stage discharge in curvilinear co-ordinates is derived from the momentum equation and the flow continuity equation. Two shear stress terms in the control equation (Equation 3) are ignored due to their negligible contributions. The impact of this simplification on the prediction is further discussed, and the corresponding error is found to be acceptable. In addition, simple analytical solutions are presented with the effect of secondary flows.

- Several cases from the published literature are selected to verify this model. The results demonstrate that the proposed model is capable of predicting the stage discharges in the meandering channel, even if the sub-area discharge is unknown

- Finally, the velocity parameter is further discussed and associated with many factors such that its initial calibration is important in a new channel. Once this calibration is 
completed, this method can predict discharges at other flow depths for that same geometry.

\section{Acknowledgements}

This work is supported by the National Natural Science Foundation of China (Grant Nos. 51479128, 51279117), the Program for New Century Excellent Talents in University of China (NCET-13-0393) and the National Science and Technology Ministry (No. 2012BAB05B02).

\section{REFERENCES}

Darby SE and Thorne CR (1996) Predicting stage-discharge curves in channels with bank vegetation. Journal of Hydraulic Engineering 122(10): 583-586.

Ervine DA, Willetts BB, Sellin RHJ and Lorena M (1993) Factors affecting conveyance in meandering compound flows. Journal of Hydraulic Engineering 119(12): 1383-1399.

Ervine DA, Babaeyan-Koopaei K and Sellin RHJ (2000) Two-dimensional solution for straight and meandering overbank flows. Journal of Hydraulic Engineering 126(9): 653-669.

Greenhill RK and Sellin RHJ (1993) Development of a simple method to predict discharges in compound meandering channels. Proceedings of the Institution of Civil Engineers Water Maritime and Energy 101(1): 37-44.

Guo Y, Zhang L, Shen Y and Zhang J (2008) Modeling study of free overfall in a rectangular channel with strip roughness. Journal of Hydraulic Engineering 134(5): 664-667.

Huai W, Xu Z, Yang Z and Zeng Y (2008) Two dimensional analytical solution for a partially vegetated compound channel flow. Applied Mathematics and Mechanics 29(8): 1077-1084.

Huai W, Gao M, Zeng Y and Li D (2009) Two-dimensional analytical solution for compound channel flows with vegetated floodplains. Applied Mathematics and Mechanics 30(9): 1121-1130.

Jing H, Guo Y, Li C and Zhang J (2009) Three-dimensional numerical simulation of compound meandering open channel flow by the Reynolds stress model. International Journal for Numerical Methods in Fluids 59(8): 927-943.

Knight DW, Omran M and Tang X (2007) Modeling depthaveraged velocity and boundary shear in trapezoidal channels with secondary flows. Journal of Hydraulic Engineering 133(1): 39-47.

Knight DW and Demetriou JD (1983) Flood plain and main channel flow interaction. Journal of Hydraulic Engineering 109(8): 1073-1092.

Lambert MF and Sellin RHJ (1996) Velocity distribution in a large-scale model of a doubly meandering compound river channel. Proceedings of the Institution of Civil Engineers Water Maritime and Energy 118(1): 10-20.
Liu C, Wright N, Liu X and Yang K (2014a) An analytical model for lateral depth-averaged velocity distributions along a meander in curved compound channels. Advances in Water Resources 7426-43.

Liu C, Liu X and Yang K (2014b) Predictive model for stagedischarge curve in compound channels with vegetated floodplains. Applied Mathematics and Mechanics 35(12): 1495-1508.

Lyness JF, Myers WRC, Cassells JBC and O'Sullivan JJ (2001) The influence of planform on flow resistance in mobile bed compound channels. Proceedings of the Institution of Civil Engineers - Water and Maritime Engineering 148(1): 5-14.

McGahey C and Samuels PG (2003) Methodology for conveyance estimation in two-stage straight, skewed and meandering channels. Proceedings of the 30th Congress of the International Association for Hydraulic Research, Thessaloniki, Greece, PP. 33-40.

Morvan H, Pender G, Wright NG and Ervine DA (2002) Threedimensional hydrodynamics of meandering compound channels. Journal of Hydraulic Engineering 128(7): 674-682.

Patra KC and Kar SK (2000) Flow interaction of meandering river with floodplains. Journal of Hydraulic Engineering 126(8): 593-604.

Patra KC, Kar SK and Bhattacharya AK (2004) Flow and velocity distribution in meandering compound channels. Journal of Hydraulic Engineering 130(5): 398-411.

Rameshwaran P and Shiono K (2007) Quasi two-dimensional model for straight overbank flows through emergent. Journal of Hydraulic Research 45(3): 302-315.

Schlichting H (1968) Boundary-layer Theory. McGraw-Hill, New York, NY, USA.

Shao X, Wang H and Chen Z (2003) Numerical modeling of turbulent flow in curved channels of compound cross-section. Advances in Water Resources 26(5): $525-539$.

Shiono K and Knight DW (1991) Turbulent open-channel flows with variable depth across the channel. Journal of Fluid Mechanics 222: 617-646.

Shiono K and Muto Y (1998) Complex flow mechanisms in compound meandering channels with overbank flow. Journal of Fluid Mechanics 376: 221-261.

Shiono K, Al-Romaih JS and Knight DW (1999) Stage-discharge assessment in compound meandering channels. Journal of Hydraulic Engineering 125(1): 66-77.

Shiono K, Chan TL, Spooner J, Rameshwaran P and Chandler JH (2009) The effect of floodplain roughness on flow structures, bedforms and sediment transport rates in meandering channels with overbank flows: part I. Journal of hydraulic research 47(1): 5-19.

Shiono K, Spooner J, Chan T, Rameshwaran P and Chandler J (2008) Flow characteristics in meandering channels with non-mobile and mobile beds for overbank flows. Journal of Hydraulic Research 46(1): 113-132. 
Water Management

Volume 169 Issue WM1
Method for assessing discharge in

meandering compound channels

Liu, Shan, Liu and Yang
Spooner J (2001) Flow Structures in a Compound Meandering Channel with Flat and Natural Bedforms. PhD dissertation, Loughborough University, Loughborough, UK

Spooner J and Shiono K (2003) Modelling of meandering channels for overbank flow. Proceedings of the Institution of Civil Engineers - Water and Maritime Engineering 156(3): 225-233.

Zarrati AR, Tamai N and Jin YC (2005) Mathematical modeling of meandering channels with a generalized depth averaged model. Journal of Hydraulic Engineering 131(6): 467-475.

\section{WHAT DO YOU THINK?}

To discuss this paper, please email up to 500 words to the editor at journals@ice.org.uk. Your contribution will be forwarded to the author(s) for a reply and, if considered appropriate by the editorial panel, will be published as discussion in a future issue of the journal.

Proceedings journals rely entirely on contributions sent in by civil engineering professionals, academics and students. Papers should be 2000-5000 words long (briefing papers should be 1000-2000 words long), with adequate illustrations and references. You can submit your paper online via www.icevirtuallibrary.com/content/journals, where you will also find detailed author guidelines. 